Running title: Signatures of selection for cattle behavior

\title{
DETECTION OF SELECTION SIGNATURES FOR AGONISTIC BEHAVIOR IN CATTLE
}

Paulina G. Eusebi ${ }^{1}$, Cortés O. ${ }^{2}$, Carleos $\mathrm{C}^{3}$, Dunner S. ${ }^{2}$, Cañón J. ${ }^{2}$

${ }^{1}$ Departament de Ciència Animal i dels Aliments, Universitat Autònoma de Barcelona, Barcelona, Spain

${ }^{2}$ Departamento de Producción Animal, Facultad de Veterinaria, Universidad Complutense de Madrid, Spain

${ }^{3}$ Departamento de Estadística e Investigación Operativa. Universidad de Oviedo. Oviedo, Spain

\section{Correspondence}

Paulina G. Eusebi, Departamento de Producción Animal, Facultad de Veterinaria, Universidad Complutense, Avenida Puerta de Hierro, s/n, 28040 Madrid, Spain.

Tel.: +34913943757

E-mail: paulinaeusebi@gmail.com 


\begin{abstract}
The identification of genomic regions including signatures of selection produced by domestication and its subsequent artificial selection processes allow the understanding of the evolution of bovine breeds. Although several studies describe the genomic variability among meat or milk production cattle breeds, there are limited publications (analysis?) orientated towards bovine behavioral features. The current study is focused on mapping genomic signatures of selection which may provide insights of differentiation between neutral and selected polymorphisms. Their effects are studied in the Lidia cattle traditionally selected for agonistic behavior compared to Spanish breeds showing tamed behavior. Two different approaches, Bayescan and SelEstim, were applied using genotypic 50K SNP Beadchip data.
\end{abstract}

Both procedures detected two genomic regions bearing genes previously related to behavioral traits. The frequencies of the selected allele in these two regions in Lidia breed were opposite to those found in the tamed breeds. In these genomic regions several putative genes associated to enriched metabolic pathways related to the behavioral development were identified, as Neurochondrin gene (NCDN) or Glutamate Ionotropic Receptor Kainate Type Subunit 3 (GRIK3) both located at BTA3 or Leucine Rich Repeat and Ig Domain Containing 2 (LINGO2) and Phospholipase A2 Activating Protein (PLAA) at BTA8.

Keywords selective sweep, aggressive behavior, Lidia breed, Spanish cattle Introduction

Since their domestication ( 10,000 years ago), cattle populations have been subjected to natural and artificial selection processes (Ajmone-Marsan et al., 2010). 
Currently most domesticated bovine breeds are specialized in milk and meat traits, and on a smaller scale in other economic traits of interest, such as leather or draft among others (Felius et al., 2014).

An early prerequisite of the domestication process in all the farm animals was probably to reduce their fear to humans (Belyaev et al., 1985). Thus, as a consequence of domestication, humans have modified the wild nature and social behavior of bovines (Jensen, 2006).

Different studies have analyzed genomic changes produced by the long-term selection in most commercial bovine breeds (Pritchard et al., 2010; Randhawa, 2016). As a consequence, several strong genomic signatures or hard-sweeps belonging to traditional selected morphological traits (muscular hypertrophy, coat color, presence/absence horns) have been reported ( Druet et al., 2013; Ramey et al., 2013, González-Rodriguez et al., 2017). But so far, studies of selection signatures focused on behavioral features are limited. The Lidia bovine breed has been selected for centuries for its agonistic- aggressive behavior by means of a series of traits registered by the breeders on a categorical scale that classifies their aggression and fighting capacity (Silva et al., 2006). Furthermore, these traits have evidenced significant heritability values, which make them suitable to genetic selection (Silva et al., 2006; MenéndezBuxadera et al., 2017,). Unlike other bovine breeds in which aggression is an undesirable trait, it is likely that the aggressiveness selection process in the Lidia bovine breed has left detectable genomic signatures (Ackey et al., 2002).

The detection of selective sweeps in quantitative traits still presents some limitations because many of the characters of interest, such as behavioral traits, are polygenic. In this case, the response to selection would be generated by modest allele 
frequency shifts at many loci, detection of which can be difficult to accomplish (Pritchard et al., 2010).

The imputation of genotypes using high-density genotyping platforms has favored the identification of genomic selection signatures using demographic models, selections models or a combination of both (Ma et al., 2015). Under selection pressure, a new genetic variant at the genomic level may show one or more of the following features: extreme allele frequencies, excess of homozygotes, high frequency of long haplotypes and/or a higher genetic differentiation among populations (Qanbari and Simianer, 2014; Randhawa, 2016).

Several selection signature detection methods are based on allele frequencies differences among populations that may simply be identified in the extreme tails of the $F_{S T}$ estimates distribution. Theoretically, loci under selection pressure or balancing selection are expected to evidence high and low levels of differentiation among populations respectively. Foll and Gaggiotti (2008) extended this approach and directly estimate the probability that each locus is subject to selection using a Bayesian method that evidenced their robustness under different demographic scenarios. However, one criticism of this kind of methodologies is that they do not quantify the intensity of selection. Recently Vitalis et al. (2014) developed a Bayesian method which allows distinguishing between selected and nearly neutral polymorphisms and estimate the intensity of selection under a genetic model that assumes the subdivision of a population into sub-populations that may exchange migrants.

In the present study, information provided by a panel of SNPs was used to analyze three groups of the Lidia bovine breed traditionally selected for agonistic related traits, and two non-specialized tamed Spanish breeds (Asturiana de los Valles 
and Morenas Gallegas) as a reference to locate genomic regions associated with agonistic traits. A marginal second objective was to identify putative candidate genes mapping within these genomic regions in order to understand the evolutionary mechanisms of the Lidia breed.

\section{Material}

A total of 213 (48 from Mexico and 165 from Spain) Lidia bovine breed individuals were genotyped using the Bovine $50 \mathrm{~K}$ SNP BeadChip (http://www.illumina.com). According to Silva et al (2006) who evidenced differences among the three main behavior characteristics that are traditionally scored in the Lidia breed (aggressiveness, ferocity and mobility), 100 samples belonging to those Spanish lineages with higher agonist behavior (SPA+) and 65 with the lower ones (SPA-) were selected to be genotyped. Those lineages with the higher and lower behavior scores also evidenced the higher genetic differentiation among the Lidia breed lineages (Cañón et al., 2008).In addition, animals from Asturiana de los Valles and from Morenas Gallegas bovine breeds were genotyped as reference group in which agonist behavior is not desirable: 60 unrelated (based on genealogical information) Asturiana de los Valles breed individuals ( 35 genotyped with the 50k BeadChip and 25 with the 777k BeadChip) and 30 individuals from the Morenas Gallegas breed genotyped with the 50k BeadChip.

The SNPs in common between the $50 \mathrm{~K}$ and $777 \mathrm{~K}$ chips were identified (Nicolazzi et al., 2015). Then, the datasets of the five groups were combined using PLINK v. 1.07 (Purcell et al., 2009) and the following SNP edits were applied including 
the removal of individuals with a call rate $<80 \%$, non-autosomal SNPs and SNPs with minor allele frequency $<0.01$. After edits, 38,577 SNPs on 303 individuals remained.

\section{Methods}

SelEstim procedure proposed by Vitalis et al. (2014) is a hierarchical Bayesian method whose model is a diffusion approximation for the distribution of allele frequency in a population subdivided into a number of groups that exchange migrants with a rate equal to $m$. This procedure provides two parameters of differentiation between groups: $\sigma$ is an average effect of selection and is a hyper-parameter that summarizes the strength of dispersion among groups at each specific locus, and the Kullback-Leiber divergence (KLD) which is a non-symmetric measure of difference between two probability distributions calculating the distance of the posterior distribution of $\sigma$ of the centering distribution. The KLD parameter represents the neutral demographic history of the groups and, KLD values are strong correlated with FST estimates. Also, an estimate of the migration rate among the breeds is provided, this parameter is scaled by the effective size (i.e. $M j=4 N j m j$ ) where $M j$ is the scaled migration parameter in the $j^{\text {th }}$ population, $N$ is the number of diploid individuals, and receives immigrants for the whole population at a rate $m$.

A first computation is performed on the whole-dataset to estimate the posterior distribution of the parameters, obtaining a "pseudo-observed" dataset; in order to provide a criterion to discriminate between neutral and selected markers, the calibration computation was then performed to achieve the thresholds $(0.95,0.99$, 
0.995 and 0.9999) quantiles of a "centering" Kullback-Leibler divergence (KLD)

empirical distribution computed from the pseudo-observed dataset.

Identification of genomic regions with selection signatures

Assuming that behavioral traits are polygenic, low influence of many loci is expected. Hence, a slide window of $\sim 10 \mathrm{MB}$ that contains each of the SNP with KLD higher than $99.99 \%$ was selected to identify genomic regions with selection signatures. Furthermore, the previously defined SNPs with KLD higher than $95 \%$ in the $10 \mathrm{Mb}$ windows were counted and used to define regions of genomic selection signatures. Gene annotation was performed by exploiting the knowledge on UMD3.1 locations of genes from the NCBI

(ftp://ftp.ncbi.nih.gov/genomes/Bos_taurus/mapview/seq_gene.md.gz), and as annotation of the bovine genome is still incomplete, BioMart from Ensembl archive release 90 (www.ensembl.org/biomart) was used to determine the orthologous human gene ID for each gene detected.

BayeScan software (Foll and Gaggiotti, 2008) was also used to detect signatures of selection, with the difference that this methodology detects divergence selection from Bayesian binomial frameworks identifying loci under selection when they show $F_{S T}$ coefficients that are significantly different to that expected under neutrality.

With BayeScan, $F_{S T}$ coefficients are split into a population-specific component $(\beta)$, common to all loci, and a locus-specific component $(\alpha)$ shared by all the populations using a logistic regression. Allele frequencies are assumed to follow a Dirichlet distribution. Selection is detected when $\alpha$ is significantly different to zero i.e. the locus-specific component is necessary to explain the observed pattern of diversity. 
When $\alpha>0$ it is assumed that directional selection is acting on the locus under analysis, while $\alpha<0$ suggests balancing or purifying selection (Foll, 2012).

Identification of genomic regions with selection signatures

The standard PLINK files were converted to BayeScan format with the PGDSpider v 2.0.7.3 software (Lischer and Excoffier, 2012) and used the same parameters set with SelEstim to perform the analyses. A first filter was applied to the results, setting a significance threshold of 5\% False Discovery Rate (Randhawa et al., 2016), and then, selecting the SNPs with alpha $(\alpha)$ values higher than 1 , as it indicates strong evidence of diversifying selection according to Jeffrey's interpretation (Foll, 2012).

\section{Results}

SelEstim

A total number of 19,287 SNPs had KLD estimates over the $50 \%$ quantile: 3,857 (90\%), 1,918 (95\%), 386 (99\%), 194 (99.5\%) and 5 (99.99\%). The genomic regions of positive selection containing at least one SNP in the last percentile and the remaining in the $95 \%$ are described in Table 1 . The migration rates $\left(M_{j}\right)$ ranged from 20.92 in the Asturiana de los Valles breed to 2.52 in the Mexican Lidia group (Table 2). Bayescan

A total of 249 outlier loci displayed strong signals of positive selection, $\alpha>1$ and q value $5 \%$ (Supplementary Table 2). A q-value of $5 \%$ means that it is expected that $5 \%$ of the outlier markers (those with a q-value $>5 \%$ ) are false positives (Foll, 2012) and therefore were discarded. 
Positional coincidences with SelEstim and Bayescan were identified in chromosome 3 and 8 (Table 3, Figure S1). Furthermore, these selective sweeps with genomic signals of positive selection were analyzed more thoroughly in order to identify candidate genes that could have been modified by selection.

\section{Selection signature at BTA3}

The pattern of the average values of selected alleles $\left(\kappa_{i j}\right)$ shown in Figure 1 evidenced that most of the polymorphisms are positively selected in the bovine populations. However, all the groups show an outlier allele at nucleotide BTA3:110,766,510 with the highest KLD value (Table 1). At this locus the intensity of selection ( $\sigma$ ) estimated, which allows for the identification of the strongest selection coefficients, had the same selection direction in the Lidia breed sub-populations and the opposite in the tamed Morenas Gallegas bovine breed. This genomic region contains several genes related to different pathways, such as the serotonergic and dopaminergic signaling pathways, which contribute in the process of differentiation in a selection oriented for behavioral related traits.

This SNP with the highest KDL value is located proximate to the Neurochondrin gene (NCDN) (BTA3: 110,784,499-110,793,283). This gene is highly expressed in the central nervous system (Supplementary Table 3) and works as a negative regulator of the $\mathrm{Ca} 2+/$ calmodulin-dependent protein kinase II (CaMKII), a key enzyme present in the early stages of memory formation and involved also in the hippocampal synaptic plasticity (Shinozaki et al., 1997; Dateki et al., 2005). This gene is highly associated with the serotonergic signaling pathway in modulating the acquisition and consolidation of memory (Moyano et al., 2004). 
The Glutamate Ionotropic Receptor Kainate Type Subunit 3 (GRIK3) gene is located close to the NCDN gene and has been identified previously by Qanabari and Simianer (2014) as candidate gene for signatures of selection in cattle. The GRIK3 gene is highly expressed in the central nervous system and is included in a QTL described in the reward-related processes underlying learning and memory (Minelli et al., 2009). Furthermore, the Disc Large Associated Protein 3 (DLGAP3) gene located within the same region is also associated in learning processes (Kähne et al., 2016).

Besides, Thyroid Hormone Receptor Associated Protein 3 (THRAP3) and Splicing Factor Proline and Glutamine Rich (SFPQ) genes, also located within the frame of this genomic region, are linked to the circadian cycle. Other genes are associated in processes implicated in domestication-related changes like sensory perception (GJB4, SAG and TRPM8), brain development and neurobehavioral functioning (POU3F1), muscle contraction (FHL3) and pigmentation (NCDN) (Xing et al. 2006; Ryu et al. 2007) (Supplementary Table 3).

Selection signature at BTA8

The pattern of the average values of the selected alleles $\left(\kappa_{i j}\right)$ revealed opposite direction of selection intensity $(\sigma)$ in the Lidia breed sub-populations compared to Asturiana de los Valles and Morenas Gallegas tamed breeds (Figure 2). Also it should be noted that the SNP with the strongest intensity of selection is present in the Lidia with higher agonist behavior (SPA+) group. Several genes are located in this genomic region, however the Leucine Rich Repeat And Ig Domain Containing 2 (LINGO2) and Phospholipase A2 Activating Protein (PLAA) genes are related with extreme 
neurobehavioral phenotypes and psychiatric disorders and probably with behavior characteristics (Supplementary Table 3).

\section{Discussion}

In the present study two Bayesian approaches that are able to detect both recent and old selection events, Bayescan and SelEstim, were applied to identify genome-wide signatures of selection in three bovine breeds traditionally selected for opposite behavior characteristics.

Additionally, SelEstim procedure also estimates intensity and direction of the selection at each locus for each population and the migration rate $\left(M_{i}\right)$ reflecting the relative admixture of each group with respect to all the groups.The relative genetic proximity of the Asturiana de los Valles breed respect to the rest of cattle populations analyzed (Table 2) is noteworthy. A similar result for the Asturiana de los Valles breed was also obtained by González Rodríguez et al. (2017) using seven Spanish bovine beef breeds, suggesting that this breed has been used as terminal sire line and crossbred individuals are introduced into the receptor populations. However, it is difficult to embrace this argument in our case taking into account the presence of the Lidia breed, which is extremely isolated and with low effective population sizes (Cortés et al., 2014).

A curious appreciation is the need to decrease the threshold of KLD to $90 \%$ to identify genomic regions under selection that are known to be under positive selection, such as the one bearing MSTN or myostatin gene (Supplementary Table 4). This threshold identified 3,857 SNPs, so this large amount of polymorphisms may be related to polygenic selection or adaptation processes (Pritchard et al., 2010), involving 
several genes or polymorphisms with minor effects. However, when the most restrictive threshold (99.99\%) was applied, the number of selected polymorphisms was reduced to only five (Table 1 ).

The difficulty to detect selective sweeps with statistical significance in polygenic traits, in which many loci shift their frequency moderately (Pritchard et al., 2010), could explain that only two genomic regions were shared with both methodologies. Other reasons may be the limitations of the 50K chip and the sample size of the analysis.

Furthermore, a high rate of false positives is expected due to the divergence in allelic frequencies between breeds (and groups within the Lidia breed and Morenas Gallegas) as a consequence of the genetic drift and founder effects; this is particularly important during the development of the cattle breeds' (Petersen et al., 2013). These factors can bias the footprints left in the genome by selection and hamper the identification of selective sweeps.

The results of the present study suggest that the methods employed are able to detect signals of selection generated by recent selection events within populations. Furthermore, the absence of regions with strong signals of selection may be hidden considering that, i) artificial selection processes do not always leave relevant signatures of selection, ii) the polygenic nature of the behavioral traits (Kemper et al., 2014) and iii) the limitations of the bovine genomic resources of the SNP Beadchip already mentioned. However both methodologies detected genomic signatures of selection in BTA3 and BTA8 regions, where genes whose higher expression is detected mainly in the prefrontal cortex of the brain, where the reactions of violence and social aggression takes place (Grafman et al., 1996; Lotze et al., 2007) (Table S3). 
Besides, the candidate genes NCDN, GRIK3, DLGAP3, THRAP3 and SFPQ located in the selective sweep at chromosome 3 are involved in the serotonergic signaling pathway involved with the development of personality and behavioral traits (Minelli et al., 2009) and also in the development of different aggressive behavior manifestations, such as fear-induced aggression (Popova et al., 2005), inter-male aggression (Kulikov and Popova, 1996; Kulikov et al., 2005; ), predatory aggression (Nikulina and Popova, 1988) and maternal aggression (da Veiga et al.,2011). However, the candidate-gene approach has mainly been conducted using rats, albeit with limited success. Studies involving putative behavioral genes such like those involved on serotonergic, catecholaminergic and glutamatergic pathways have failed to find variants of significance, mainly because of a small number of study subjects and a lack of functional assays (Spady and Ostrander, 2008).

In conclusion, the present study identifies two genomic regions associated with agonistic related traits in cattle. The direction of selection of both regions differed between the aggressive Lidia breed and the tamed Asturiana de los Valles and Morenas gallegas breeds that were used for comparative purposes. These findings corroborate that intensive targeted selection for different goal traits have left detectable imprints in the genome.

\section{Conflict of interest statement}

All authors declare that they have no conflict of interest.

\section{Acknowledgements}

The authors gratefully acknowledge to the Breeders of the ANCTL, to the Federación de razas autóctonas gallegas-BOAGA and to the Asociación Española de 
criadores de ganado vacuno selecto de la raza Asturiana de los Valles- ASEAVA for

providing the animal material. This work has been funded by grants of CONACYT and

CONARGEN from the Mexican government and by the Genetics Laboratory of the

Animal Production Department at the Universidad Complutense of Madrid.

\section{References}

Ajmone-Marsan P., Garcia J. F., \& Lenstra J. A. (2010). On the origin of cattle: how aurochs became cattle and colonized the world. Evol. Anthropol., 19(4), 148-157.

Akey J. M., Zhang G., Zhang K., Jin, L., \& Shriver M. D. (2002). Interrogating a highdensity SNP map for signatures of natural selection. Gen. Res., 12(12), 1805-1814.

Belyaev D.K., Plyusnina I.Z. \& Trut .LN. (1985) Domestication in the silver fox (Vulpes fulvus Desm): Changes in physiological boundaries of the sensitive period of primary socialization. Appl. Anim. Behav. Sci., 13:359-370.

Cañón J., Tupac-Yupanqui I., García-Atance M. A., Cortés O., García D., Fernández J., \& Dunner S. (2008). Genetic variation within the Lidia bovine breed. Anim. Genet., 39(4), 439-445.

Cortés, O., Sevane, N., Baro, J.A., Cañón, J., (2014). Pedigree analysis of a highly fragmented population, the Lidia cattle breed. Livest. Sci., 167:1-8.

Dateki M., Horii T., Kasuya Y., Mochizuk, R., Nagao Y., Ishida J., Sugiyama F., Tanimoto K., Yagami K., Imai Hi. \& Fukamizu, A. (2005). Neurochondrin negatively regulates CaMKII phosphorylation, and nervous system-specific gene disruption results in epileptic seizure. J. Biol. Chem., 280(21), 20503-20508.

da Veiga C.P., Miczek K.A., Lucion A.B. \& de Almeida RM (2011) Social instigation and aggression in postpartum female rats: role of $5-\mathrm{Ht} 1 \mathrm{~A}$ and $5-\mathrm{Ht} 1 \mathrm{~B}$ receptors in the dorsal raphe nucleus and prefrontal cortex. Psychopharmacology 213:475-487.

Druet, T., Pérez-Pardal, L., Charlier, C., \& Gautier, M. (2013). Identification of large selective sweeps associated with major genes in cattle. Anim. Genet. , 44(6), 758-762.

Eusebi P.G., Cortés O., Dunner S. and Cañón J. (2017) Genomic diversity and population structure of Mexican and Spanish bovine Lidia breed. Anim.Genet., 48(6), 682-685.

Felius M., Beerling M. L., Buchanan D. S., Theunissen B., Koolmees P. A., Lenstra J. A. (2014) On the history of cattle genetic resources. Diversity, 6(4), 705-750. 
Foll M. \& Gaggiotti O. E. (2008) A genome scan method to identify selected loci appropriate for both dominant and codominant markers: A Bayesian perspective. Genetics, 180, 977-93.

Foll M. (2012). BayeScan v2. 1 user manual. Ecology, 20, 1450-1462.

González-Rodríguez A., Munilla S., Mouresan E. F., Cañas-Álvarez J. J., Día, C., Piedrafita J., Altarriba J., Baro J.A., Molina A. \& Varona, L. (2016). On the performance of tests for the detection of signatures of selection: a case study with the Spanish autochthonous beef cattle populations. Gen. Sel. Evol., 48(1), 81.

González-Rodríguez A., Munilla S., Mouresan E. F., Cañas-Álvarez J. J., Baro J. A., Molina A., \& Varona L. (2017). Genomic differentiation between Asturiana de los Valles, Avileña-Negra Ibérica, Bruna dels Pirineus, Morucha, Pirenaica, Retinta and Rubia Gallega cattle breeds. Animal, 1-13.

Grafman J., Schwab K., Warden D., Pridgen A., Brown H. R. \& Salazar A. M. (1996). Frontal lobe injuries, violence, and aggression a report of the vietnam head injury study. Neurology, 46(5), 1231-1231.

Jensen, P. (2006). Domestication-from behavior to genes and back again. Applied Anim.l Behav. Sci. 97(1), 3-15.

Kähne T., Richter S., Kolodziej A., Smalla K. H., Pielo, R., Engler A., Ohl F.W.,. Dieterich D.C., Tischmeyer W., Naumann M., \& Naumann M. (2016). Proteome rearrangements after auditory learning: high-resolution profiling of synapse-enriched protein fractions from mouse brain. J. Neuroch, 138(1), 124-138.

Kemper K.E., Saxton S.J., Bolormaa S., Hayes B.J. \& Goddard M.E. (2014) Selection for complex traits leaves little or no classic signatures of selection. BMC Genomics.,15:246.

Kulikov A.V. \& Popova N.K. (1996) Association between intermale aggression and genetically defined tryptophan hydroxylase activity in the mouse brain. Aggressive Behavior., 22:111-117.

Kulikov A.V., Osipova D.V., Naumenko V.S. \& Popova N.K. (2005) Association between Tph2 gene polymorphism, brain tryptophan hydroxylase activity and aggressiveness in mouse strains. Genes. Brain. Behav., 4:482-485.

Lischer H. E. L. \& Excoffier, L. (2012).PGDSpider: An automated data conversion tool for connecting population genetics and genomics programs. Bioinformatics, 28, 298-99.

Lotze M., Veit R., Anders S. \& Birbaumer, N. (2007). Evidence for a different role of the ventral and dorsal medial prefrontal cortex for social reactive aggression: An interactive fMRI study. Neuroimage, 34(1), 470-478. 
Ma Y., Ding X., Qanbari S., Weigend S., Zhang Q., \& Simianer H. (2015). Properties of different selection signature statistics and a new strategy for combining them. Heredity., 115(5), 426.

Makina S. O., Muchadeyi F. C., Marle-Köster E., Taylor J. F., Makgahlela M. L., \& Maiwashe A. (2015). Genome-wide scan for selection signatures in six cattle breeds in South Africa. Gen. Sel. Evol., 47(1), 92.

Menéndez-Buxadera A., Cortés O., \& Cañon J. (2017). Genetic (co) variance and plasticity of behavioural traits in Lidia bovine breed. Ital. J. Anim. Sci., 1-9.

Minelli A., Scassellati C., Bonvicin, C., Perez J., \& Gennarelli M. (2009). An association of GRIK3 Ser310Ala functional polymorphism with personality traits. Neuropsychobiology, 59(1), 28-33.

Moyano S., Del Río J. \& Frechilla, D. (2004). Role of hippocampal CaMKII in serotonin 5HT1A receptor-mediated learning deficit in rats. Neuropsychopharmacology, 29(12), 2216.

Nicolazzi E. L., Caprera A., Nazzicari N., Cozzi P., Strozzi F., Lawley C.. Pirani A., Chandrasen S., Brew F., Jorjani H., \& Evans, G. (2015). SNPchiMp v. 3: integrating and standardizing single nucleotide polymorphism data for livestock species. BMC genomics., 16(1), 1.

Nikulina E.M. \& Popova N.K. (1988) Predatory aggression in the mink (Mustela vison): roles of serotonin and food satiation. Aggressive Behavior 14:77-84.

Petersen J. L., Mickelson J. R., Rendahl A. K., Valberg S. J., Andersson L. S., Axelsson J.,Bailey E., Bannasch D., Binns M.M., Borges A.S. \& Brama, P. (2013). Genome-wide analysis reveals selection for important traits in domestic horse breeds. PLoS genetics, 9(1), e1003211.

Popova N.K., Naumenko V.S., Plyusnina I.Z. \& Kulikov A.V. (2005) Reduction in 5-HT1A receptor density, 5-HT1A mRNA expression, and functional correlates for 5-HT1A receptors in genetically defined aggressive rats. J. Neurosci. Res. 80:286-292.

Pritchard J. K., Pickrell J. K., \& Coop G. (2010). The genetics of human adaptation: hard sweeps, soft sweeps, and polygenic adaptation. Curr. Biol., 20(4), R208-R215.

Purcell, S., Neale, B., Todd-Brown et al. (2007). PLINK: a tool set for whole-genome association and population-based linkage analyses.Am. J. Hum. Genet., 81(3), 559-575.

Qanbari, S., \& Simianer, H. (2014). Mapping signatures of positive selection in the genome of livestock. Livest. Sci., 166, 133-143.

Ramey H. R., Decker J. E., McKay S. D., Rolf M. M., Schnabel R. D., \& Taylor J. F. (2013). Detection of selective sweeps in cattle using genome-wide SNP data. BMC genomics., 14(1), 382. 
Shinozaki K., Maruyama K., Kume H., Kuzume H. \& Obata K. (1997). A novel brain gene, norbin, induced by treatment of tetraethylammonium in rat hippocampal slice and accompanied with neurite-outgrowth in neuro 2a cells. Biochem.Biophys. Res. Commun., 240(3), 766-771.

Silva B., Gonzalo A., \& Cañón J. (2006). Genetic parameters of aggressiveness, ferocity and mobility in the fighting bull breed. Anim. Res., 55(1), 65-70.

Spady T. C., \& Ostrander E. A. (2008). Canine behavioral genetics: pointing out the phenotypes and herding up the genes. Am. J. of Hum. Genet., 82(1), 10-18.

Vitalis R., Gautie, M., Dawson K. J., \& Beaumont M. A. (2013). Detecting and measuring selection from gene frequency data. Genetics, genetics-113.

Xing H., Ling J., Chen M., \& Gu, J. G. (2006). Chemical and cold sensitivity of two distinct populations of TRPM8-expressing somatosensory neurons. J.Neurophysiol., 95(2), 1221-1230.

Zhang C. (2010). Gap junctions in olfactory neurons modulate olfactory sensitivity. BMC Neurosci., 11(1), 108.

\section{Supporting information}

Supplementary Table 1 Classification of the Spanish lineages selected according to their extreme divergence in origins. (N) Number of individuals per lineage.

Supplementary Table 2 Selection signatures identified with the BayeScan software, with a threshold set on a $5 \%$ false discovery rate (FDR) and alpha $(\alpha)$ values $<1$. Chromosome number (Chr), Base Pair position (bp).

Supplementary Table 3 Candidate genes identified within the putative selective sweeps in the chromosome number (Chr) and at the Base pair position (bp).

Supplementary Table 4 Positional concordance between the SNPs detected at the $90 \%$ KLD quantile of SelEstim and other studies. Regions were constructed under the same selection criteria, using $10 \mathrm{Mb}$ sliding window spans. Chr: chromosome, Reg: region in $\mathrm{Mb}, \mathrm{N}$ SNP: number of markers with the higher value of KLD, and its positional concordance with previously reported regions detected on Spanish cattle breeds.

Supplementary Figure 1 Whole-genome scan of the Kullback-Leiber divergence (KLD) and the locus specific component (alpha) results obtained with the SelEstim, and Bayescan software's respectively. The gray lines indicate the thresholds of significance for each approach. Genomic coordinates and statistical significance are plotted in the $x$ - and $y$ - axis, respectively. 


\section{Tables}

Table 1 Putative selective sweeps identified with SelEstim. Chr: chromosome, N SNP: number of SNPs included in the genomic region with KLD over $95 \%$ and at least one SNP over 99.99\%, Mb: Mega base pairs, and the higher value of the Kullback-Leibler Divergence (KLD) of the SNPs included in the selective sweep. .

\begin{tabular}{cccccc}
\hline Selective sweep & Chr & N SNP & Mb Start & Mb End & Higher KLD \\
\hline 1 & 3 & 9 & 109.49 & 119.08 & 1.92 \\
2 & 8 & 8 & 14.89 & 27.98 & 1.92 \\
3 & 11 & 11 & 15.07 & 24.92 & 2.55 \\
4 & 13 & 5 & 26.52 & 31.82 & 1.98 \\
5 & 18 & 12 & 47.83 & 54.86 & 2.22 \\
\hline
\end{tabular}

Table 2 Estimate of the migration $\left(M_{j}\right)$ parameters for the five groups, mean values and standard deviations (Std. Dev.).

\begin{tabular}{lll}
\hline Group & Mean & Std. Dev. \\
\hline Asturiana de los Valles & 20.92 & 0.24 \\
Morenas Gallegas & 9.14 & 0.09 \\
Lidia Mexico & 2.52 & 0.02 \\
Lidia Spain(+) & 12.81 & 0.13 \\
Lidia Spain(-) & 4.68 & 0.04
\end{tabular}

Table 3 Genomic concordance of the selective sweeps identified with Bayescan and SelEstim approaches. Chr: chromosome, N SNP: number of markers, Mb: mega base pairs.

\begin{tabular}{c|cccc|c|c} 
Chr & \multicolumn{5}{|c|}{ SelEstim } & \multicolumn{2}{c}{ Bayescan } \\
& N SNPS & Mb Start & Mb End & $\begin{array}{c}\text { Higher } \\
\text { KLD }\end{array}$ & Mb & $\begin{array}{c}\text { Higher } \\
\boldsymbol{\alpha}\end{array}$ \\
\hline 3 & 9 & 109.49 & 119.08 & 1.92 & 116.8 & 1.02 \\
\hline 8 & 8 & 14.89 & 27.98 & 1.92 & 19.9 & 1.16 \\
\cline { 5 - 7 } & & & & & 22.3 & 1.28
\end{tabular}

\section{Figure legends}

Figure 1 Plot of the putative selective sweep localized in BTA3 between 106 and 119 $\mathrm{Mb}$. The left boxplot are the mean selection coefficient $(\sigma)$ per group and in right boxplot the mean values of the allele selected $\left(\mathrm{K}_{\mathrm{ij}}\right)$ for each group, where RAV= Asturiana de los Valles, MG= Morenas gallegas, Mex= Lidia from Mexico, Spanish(-)= Spanish Lidia less aggressive group and Spanish $(+)=$ Spanish Lidia more aggressive group.

Figure 2 Plot of the putative selective sweep localized in BTA8 between the 13 and 24 $\mathrm{Mb}$. The left boxplot are the mean selection coefficient $(\sigma)$ per group and in right 
boxplot the mean values of the allele selected $\left(\mathrm{K}_{\mathrm{ij}}\right)$ for each group, where AST= Asturiana de los Valles, MG= Morenas gallegas, Mex= Lidia from Mexico, Spanish(-)= Spanish Lidia less aggressive group and Spanish $(+)=$ Spanish Lidia more aggressive group. 


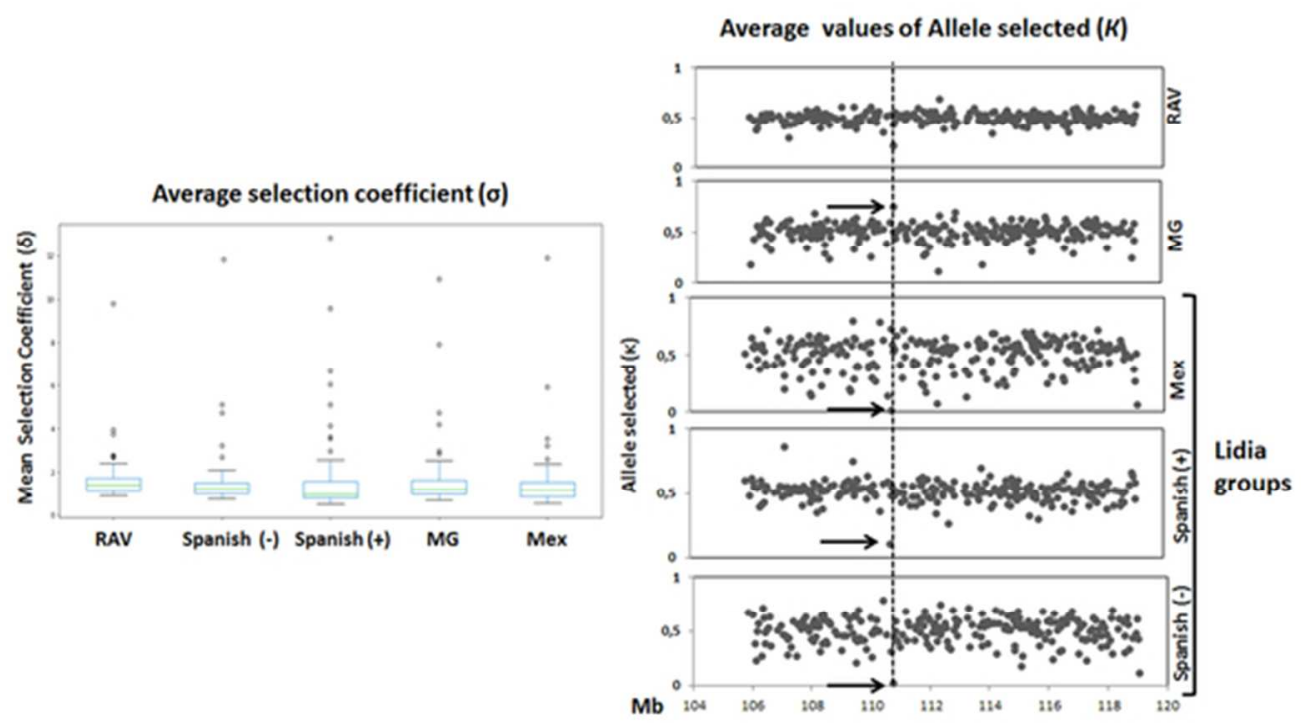

Figure 1. Plot of the putative selective sweep localized in BTA3 between 106 and $119 \mathrm{Mb}$. The left boxplot are the mean selection coefficient $(\sigma)$ per group and in right boxplot the mean values of the allele selected ( $\mathrm{kij}$ ) for each group, where RAV= Asturianas de los Valles, MG= Morenas gallegas, Mex= Lidia from Mexico, Spanish (-) = Spanish Lidia less aggressive and Spanish $(+)=$ Spanish Lidia more aggressive group.

$49 \times 28 \mathrm{~mm}(300 \times 300 \mathrm{DPI})$ 


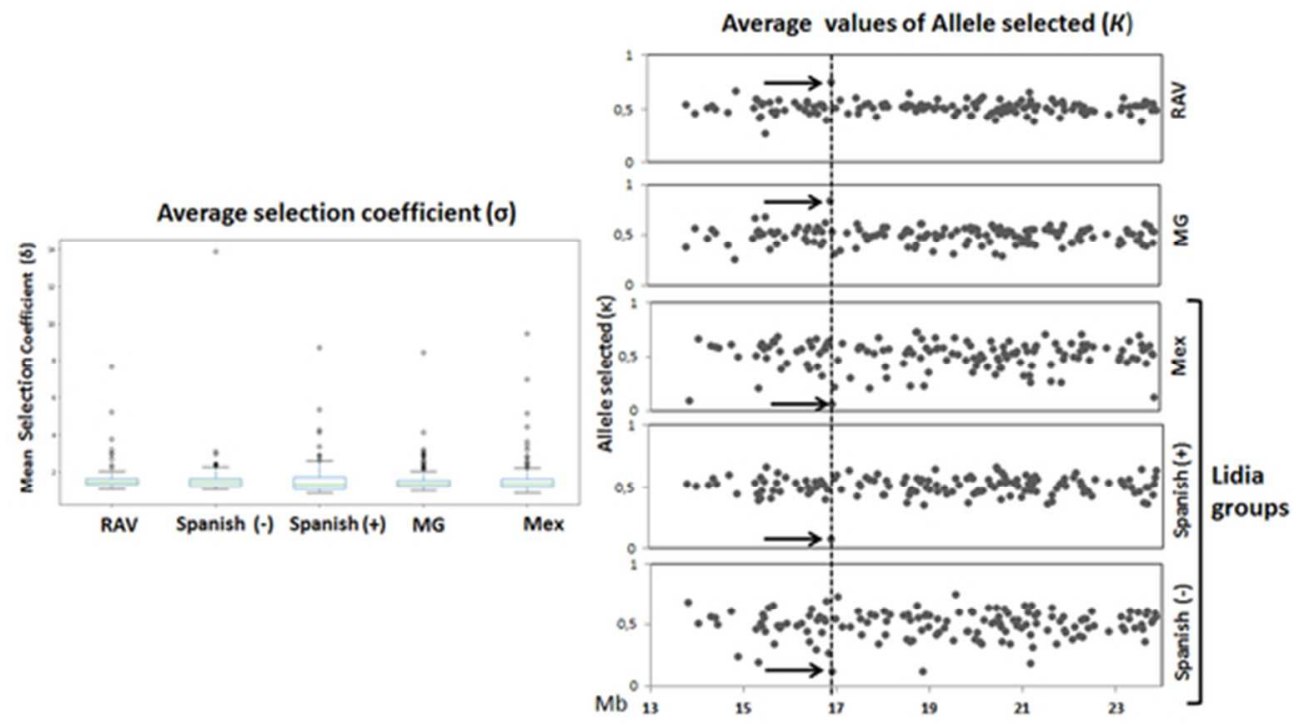

Figure 2. Plot of the putative selective sweep localized in BTA8 between the 13 and $24 \mathrm{Mb}$. The left boxplot are the mean selection coefficient $(\sigma)$ per group and in right boxplot the mean values of the allele selected ( $\mathrm{kij}$ ) for each group, where RAV= Asturianas de los Valles, MG= Morenas gallegas, Mex= Lidia from Mexico, Spanish (-) = Spanish Lidia less aggressive and Spanish $(+)=$ Spanish Lidia more aggressive group.

$49 \times 28 \mathrm{~mm}(300 \times 300 \mathrm{DPI})$ 
Supplementary Table 1 Classification of the Spanish lineages selected according to their extreme divergence in origins. (N) Number of individuals per lineage.

\begin{tabular}{lc|lc}
\hline \multicolumn{2}{c|}{ Most agonistic behavior } & \multicolumn{2}{c}{ Less agonistic behavior } \\
Name & N & Name & N \\
\hline Albaserrada & 15 & Atanasio Fernández & 14 \\
Santa Coloma & 36 & Baltasar Ibán & 12 \\
Saltillo & 15 & Conde de la Corte & 10 \\
Miura & 9 & Domecq & 29 \\
Vega-Villar & 17 & & \\
Pablo Romero & 9 & & \\
\hline
\end{tabular}

Supplementary Table 2 Selection signatures identified with the BayeScan software, with a threshold set on a $5 \%$ false discovery rate (FDR) and alpha $(\alpha)$ values $<1$.

Chromosome number (Chr), Base Pair position (bp).

\begin{tabular}{|c|c|c|c|c|}
\hline Chr & Marker & BP & q-value & $\alpha$ \\
\hline 1 & ARS-BFGL-BAC-14930 & 1782724 & 0.012 & 1.27 \\
\hline 1 & ARS-BFGL-NGS-11433 & 1851399 & 0.005 & 1.33 \\
\hline 1 & ARS-BFGL-BAC-7317 & 1983902 & 0.047 & 1.03 \\
\hline 1 & ARS-BFGL-NGS-65157 & 4348137 & 0.036 & 1.07 \\
\hline 1 & BTB-01225182 & 15109714 & 0.032 & 1.00 \\
\hline 1 & Hapmap54262-rs29018839 & 47102513 & 0.049 & 1.02 \\
\hline 1 & ARS-BFGL-NGS-25522 & 79324497 & 0.017 & 1.17 \\
\hline 1 & BTB-00034221 & 79591551 & 0.002 & 1.24 \\
\hline 1 & Hapmap47824-BTA-107197 & 82147822 & 0.046 & 1.04 \\
\hline 1 & Hapmap55716-rs29015966 & 155654021 & 0.011 & 1.25 \\
\hline 2 & ARS-BFGL-NGS-39978 & 5757355 & 0.001 & 1.32 \\
\hline 2 & ARS-BFGL-NGS-93854 & 5877768 & 0.000 & 1.79 \\
\hline 2 & ARS-BFGL-NGS-112454 & 6675045 & 0.000 & 1.36 \\
\hline 2 & Hapmap54028-rs29023584 & 6792631 & 0.000 & 1.55 \\
\hline 2 & BTB-00862033 & 8884527 & 0.004 & 1.20 \\
\hline 2 & BTB-00862061 & 8933349 & 0.026 & 1.02 \\
\hline 2 & BTB-02094616 & 8955989 & 0.025 & 1.05 \\
\hline 2 & ARS-BFGL-NGS-111530 & 9202511 & 0.000 & 1.77 \\
\hline 2 & Hapmap51202-BTA-28358 & 10216677 & 0.019 & 1.11 \\
\hline 2 & BTB-00084343 & 22644664 & 0.010 & 1.31 \\
\hline 2 & Hapmap58509-rs29024139 & 37635484 & 0.046 & 1.03 \\
\hline 2 & ARS-BFGL-NGS-3108 & 38636687 & 0.032 & 1.09 \\
\hline 2 & Hapmap48631-BTA-117251 & 47084459 & 0.013 & 1.21 \\
\hline 2 & ВTB-01165311 & 55283216 & 0.008 & 1.14 \\
\hline 2 & ARS-BFGL-NGS-114696 & 72609348 & 0.010 & 1.22 \\
\hline 2 & ARS-BFGL-NGS-112489 & 72632052 & 0.014 & 1.19 \\
\hline 2 & Hapmap51113-BTA-100127 & 89444485 & 0.001 & 1.39 \\
\hline 2 & ARS-BFGL-NGS-118468 & 89707126 & 0.002 & 1.35 \\
\hline 2 & ARS-BFGL-NGS-26540 & 89752875 & 0.016 & 1.18 \\
\hline 2 & ARS-BFGL-NGS-3488 & 92715999 & 0.028 & 1.34 \\
\hline 2 & ARS-BFGL-NGS-55602 & 93600676 & 0.014 & 1.21 \\
\hline
\end{tabular}


6 ARS-BFGL-NGS-110738

6 Hapmap23854-BTC-062412

6 Hapmap24039-BTC-031958

6 Hapmap30134-BTC-034283

$\begin{array}{lll}37252345 & 0.034 & 1.05\end{array}$

6 Hapmap33170-BTC-071249

6 Hapmap31624-BTC-046805

$\begin{array}{lll}37610880 & 0.001 & 1.38\end{array}$

$\begin{array}{lll}37704254 & 0.003 & 1.27\end{array}$

$\begin{array}{lll}38464203 & 0.000 & 1.47\end{array}$

$\begin{array}{lll}39371150 & 0.039 & 1.01\end{array}$

6 ARS-BFGL-NGS-95035

$6 \quad$ BTB-01899352

$6 \quad$ BTB-00262807

6 Hapmap53308-rs29024839

6 Hapmap23494-BTC-046433

$\begin{array}{lll}41443081 & 0.002 & 1.32\end{array}$

$\begin{array}{lll}46788536 & 0.000 & 1.39\end{array}$

$\begin{array}{lll}72969608 & 0.007 & 1.17\end{array}$

$\begin{array}{lll}73032896 & 0.007 & 1.16\end{array}$

$\begin{array}{lll}73057109 & 0.018 & 1.06\end{array}$

6 Hapmap47732-BTA-77644

$\begin{array}{lll}103307689 & 0.023 & 1.12\end{array}$

6 ARS-BFGL-NGS-30562

6 ARS-BFGL-NGS-31881

$6 \quad$ BTB-00283603

6 BTB-00280923

7 BTB-01172167

7 BTB-01398686

7 ARS-BFGL-NGS-35201

$\begin{array}{lll}103796957 & 0.019 & 1.15\end{array}$

$\begin{array}{lll}104896770 & 0.011 & 1.27\end{array}$

$\begin{array}{lll}104922823 & 0.013 & 1.21\end{array}$

$\begin{array}{lll}109719477 & 0.038 & 1.06\end{array}$

$\begin{array}{lll}110745167 & 0.021 & 1.12\end{array}$

$\begin{array}{lll}8078251 & 0.026 & 1.13\end{array}$

$\begin{array}{lll}22996615 & 0.004 & 1.27\end{array}$

7 BTB-01562090

7 ARS-BFGL-NGS-88233

$\begin{array}{lll}26494064 & 0.043 & 1.26\end{array}$

$\begin{array}{lll}39737588 & 0.001 & 1.48\end{array}$

7 ARS-BFGL-NGS-69727

$\begin{array}{lll}41718041 & 0.000 & 1.41\end{array}$

7 ARS-BFGL-NGS-3154

$\begin{array}{lll}41742027 & 0.007 & 1.25\end{array}$

7 Hapmap60894-rs29027443

$\begin{array}{lll}84520981 & 0.037 & 1.13\end{array}$

$\begin{array}{lll}90080630 & 0.000 & 1.46\end{array}$

7 Hapmap38913-BTA-80438

$\begin{array}{lll}102342232 & 0.025 & 1.03\end{array}$

7 BTA-80441-no-rs

7 BTB-01548437

8 ARS-BFGL-NGS-10448

8 ARS-BFGL-NGS-113904

$\begin{array}{lll}103779001 & 0.003 & 1.34\end{array}$

$\begin{array}{lll}107770283 & 0.000 & 1.31\end{array}$

8 Hapmap48586-BTA-106838

$\begin{array}{lll}1305820 & 0.044 & 1.03\end{array}$

$\begin{array}{lll}3617253 & 0.013 & 1.19\end{array}$

8 ARS-BFGL-NGS-22169

$\begin{array}{lll}5036995 & 0.011 & 1.32\end{array}$

$\begin{array}{lllll}8 & \text { BTB-01369338 } & 19967642 & 0.037 & 116\end{array}$

$\begin{array}{lllll}8 & \text { Hapmap41606-BTA-64576 } & 22362375 & 0.017 & 1.28\end{array}$

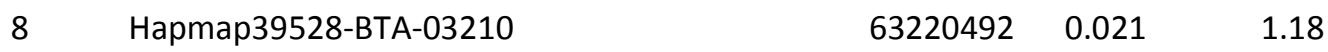

8 Hapmap57206-rs29025935

8 Hapmap34874-BES3_Contig415_1312

8 ARS-BFGL-NGS-11101

8 ARS-BFGL-NGS-97157

8 ARS-BFGL-NGS-62416

9 ARS-BFGL-NGS-14862

10 Hapmap35793-SCAFFOLD311748_23298

10 Hapmap23512-BTA-125084

10 ARS-BFGL-NGS-97105

$\begin{array}{lll}64068111 & 0.041 & 1.16\end{array}$

$\begin{array}{lll}79559282 & 0.022 & 1.04\end{array}$

$\begin{array}{lll}81108363 & 0.035 & 1.29\end{array}$

$\begin{array}{lll}90539222 & 0.023 & 1.11\end{array}$

$\begin{array}{lll}104484489 & 0.025 & 1.34\end{array}$

$\begin{array}{lll}58044059 & 0.008 & 1.27\end{array}$

$8204943 \quad 0.000 \quad 1.46$

$\begin{array}{lll}8232496 & 0.002 & 1.25\end{array}$

$\begin{array}{lll}8255705 & 0.014 & 1.18\end{array}$ 
ARS-BFGL-NGS-35518

ARS-BFGL-BAC-12994

ARS-BFGL-NGS-116708

ARS-BFGL-NGS-88133

ARS-BFGL-NGS-38003

ARS-BFGL-NGS-14399

$\begin{array}{lll}58488593 & 0.016 & 1.18\end{array}$

$\begin{array}{lll}59615280 & 0.024 & 1.15\end{array}$

$\begin{array}{lll}59667969 & 0.035 & 1.09\end{array}$

$\begin{array}{lll}62081998 & 0.015 & 1.10\end{array}$

$\begin{array}{lll}65212580 & 0.008 & 1.24\end{array}$

$\begin{array}{lll}30128818 & 0.001 & 1.33\end{array}$

$\begin{array}{lll}41854251 & 0.012 & 1.34\end{array}$

$\begin{array}{lll}79051605 & 0.003 & 1.22\end{array}$

$\begin{array}{lll}82409810 & 0.006 & 1.28\end{array}$

$\begin{array}{lll}98219856 & 0.036 & 1.06\end{array}$

BTA-92165-no-rs

BTA-115591-no-rs

ARS-BFGL-NGS-8073

ARS-BFGL-NGS-21967

Hapmap42181-BTA-31908

ARS-BFGL-NGS-116062

ARS-BFGL-NGS-19009

ARS-BFGL-NGS-21649

Hapmap24767-BTC-058058

Hapmap25450-BTC-055819

ARS-BFGL-NGS-103654

UA-IFASA-7441

BTA-107905-no-rs

ARS-BFGL-NGS-84700

BTB-01708641

Hapmap31256-BTC-012280

BTB-00573962

Hapmap40697-BTA-24633

BTB-01786632

ARS-BFGL-NGS-117317

ARS-BFGL-NGS-37606

ARS-BFGL-NGS-115927

ARS-BFGL-NGS-54901

Hapmap52590-rs29020878

ARS-BFGL-NGS-80386

BTB-00596838

ARS-BFGL-NGS-108402

$\begin{array}{lll}36335510 & 0.001 & 1.48\end{array}$

$\begin{array}{lll}51128057 & 0.015 & 1.11\end{array}$

$\begin{array}{lll}79224151 & 0.043 & 1.17\end{array}$

$\begin{array}{lll}81119950 & 0.027 & 1.19\end{array}$

$\begin{array}{lll}18762878 & 0.016 & 1.09\end{array}$

$\begin{array}{lll}25323323 & 0.031 & 1.09\end{array}$

$\begin{array}{lll}46510476 & 0.003 & 1.25\end{array}$

$\begin{array}{lll}57446360 & 0.012 & 1.31\end{array}$

$\begin{array}{lll}71271729 & 0.012 & 1.20\end{array}$

$\begin{array}{lll}9964138 & 0.025 & 1.03\end{array}$

$\begin{array}{lll}10424817 & 0.033 \quad 1.10\end{array}$

$\begin{array}{lll}17109001 & 0.045 & 1.14\end{array}$

$\begin{array}{lll}28053009 & 0.013 & 1.11\end{array}$

$\begin{array}{lll}38440957 & 0.039 & 1.06\end{array}$

$\begin{array}{lll}41809705 & 0.043 \quad 1.03\end{array}$

$\begin{array}{lll}44291954 & 0.006 & 1.20\end{array}$

$\begin{array}{lll}55910671 & 0.014 & 1.31\end{array}$

$\begin{array}{lll}59577845 & 0.003 & 1.56\end{array}$

$\begin{array}{lll}12476540 & 0.033 & 1.07\end{array}$

$\begin{array}{lll}12520480 & 0.033 & 1.08\end{array}$

$\begin{array}{lll}12567793 & 0.034 & 1.07\end{array}$

$\begin{array}{lll}26291524 & 0.016 & 1.17\end{array}$

$\begin{array}{lll}39469268 & 0.020 & 1.13\end{array}$

$\begin{array}{lll}40356568 & 0.005 & 1.33\end{array}$

$\begin{array}{lll}41383202 & 0.030 & 1.22\end{array}$

$\begin{array}{lll}43496588 & 0.022 & 1.13\end{array}$

$\begin{array}{lll}43883527 & 0.002 & 1.34\end{array}$

$\begin{array}{lll}44066727 & 0.009 & 1.32\end{array}$

$\begin{array}{lll}51853820 & 0.003 & 1.42\end{array}$

$\begin{array}{lll}52026483 & 0.002 & 1.42\end{array}$ 
$\begin{array}{lll}62823352 & 0.004 & 1.27\end{array}$

15 BTB-00611237

$\begin{array}{lll}66082534 & 0.019 & 1.22\end{array}$

15

ARS-BFGL-NGS-3375

$\begin{array}{lll}76729776 & 0.023 & 1.12\end{array}$

15

BTA-16481-no-rs

ARS-BFGL-NGS-5434

$\begin{array}{lll}81335502 & 0.000 & 1.63\end{array}$

15

ARS-BFGL-NGS-75856

81869228

1.16

16

Hapmap39562-BTA-40351

$4138735 \quad 0.018 \quad 1.25$

ARS-BFGL-NGS-31253

$\begin{array}{lll}38830470 & 0.004 & 1.39\end{array}$

16

55752131

74481728

0.042

1.04

$\begin{array}{lll}10726289 & 0.027 & 1.04\end{array}$

17

BTA-41003-no-rs

17

ARS-BFGL-NGS-18235

46325733

0.004

1.31

17

BTB-01953505

17 ARS-BFGL-BAC-32128

17 Hapmap43749-BTA-104425

17 ARS-BFGL-NGS-111330

17 ARS-BFGL-NGS-117653

21 Hapmap59281-rs29027629

$\begin{array}{lll}67904403 & 0.000 & 1.57\end{array}$ 
ARS-BFGL-NGS-113315

$\begin{array}{ll}59204394 & 0.001\end{array}$

ARS-BFGL-NGS-77507

$59237587 \quad 0.007$

1.17

23

UA-IFASA-4515

8289524

0.000

1.79

ARS-BFGL-BAC-30064

12548841

0.032

1.30

ARS-BFGL-NGS-60100

28598059

0.006

1.31

Hapmap41146-BTA-108100

$5504370 \quad 0.029$

1.24

BTB-01970944

$\begin{array}{lll}11645293 & 0.044 & 1.04\end{array}$

24

ARS-BFGL-NGS-14250

Hapmap57973-rs29024513

12916861

0.017

1.41

ARS-BFGL-NGS-100146

13837820

0.004

1.52

ARS-BFGL-NGS-1521

ARS-BFGL-NGS-117929

36464131

0.009

1.17

$41579956 \quad 0.008$

1.23

ARS-BFGL-NGS-62539

41603109

1.30

ARS-BFGL-NGS-13255

46537819

0.010

1.22

$50266999 \quad 0.004$

1.28

ARS-BFGL-NGS-111274

$\begin{array}{ll}50306117 & 0.005\end{array}$

1.27

ARS-BFGL-NGS-117573

58081600

1.05

ARS-BFGL-NGS-40549

23777990

0.045

1.48

ARS-BFGL-NGS-57317

32823036

0.001

1.02

ARS-BFGL-NGS-20432

$36309252 \quad 0.015$

1.32

ARS-BFGL-NGS-105741

39286957

0.011

1.21

ARS-BFGL-NGS-53115

11528933

0.048

1.06

Hapmap57957-rs29017349

26841116

0.047

1.11

ARS-BFGL-NGS-15144

$42933219 \quad 0.004$

1.28

Hapmap41566-BTA-52258

44181047

0.019

1.20

ARS-BFGL-NGS-14909

$44238995 \quad 0.009$

1.30

ARS-BFGL-NGS-109807

49918587

0.002

1.34

UA-IFASA-7203

22500067

1.28

Hapmap27965-BTA-148364

29300595

0.009

1.17

ARS-BFGL-NGS-112047

30945497

0.016

1.18

Hapmap57132-rs29011225

$40916876 \quad 0.012$

1.20

BTB-02050030

$18592194 \quad 0.028$

1.11

BTB-02082594

$\begin{array}{ll}21125373 & 0.022\end{array}$

1.25

ARS-BFGL-NGS-94949

$26697399 \quad 0.000$

1.42

Supplementary table $\mathbf{3}$ Candidate genes identified within the putative selective sweeps in the chromosome number (Chr) and at the Base pair position (bp).

\begin{tabular}{|c|c|c|c|c|}
\hline Chr & Position (bp)1 & Gene & Function/association & $\begin{array}{l}\text { Major expression } \\
\text { tissue (RNA-seq*) }\end{array}$ \\
\hline 3 & $109,293,456$ & GRIK3 & Reward-related learning & $\begin{array}{l}\text { Cortex, brain, tibial } \\
\text { nerve }\end{array}$ \\
\hline 3 & $111,388,593$ & DLGAP3 & Neuronal signing and learning & $\begin{array}{l}\text { Cortex, brain, } \\
\text { cerebellum }\end{array}$ \\
\hline 3 & $110,172,233$ & THRAP3 & Circadian cycle and behavior & $\begin{array}{l}\text { Brain, retina, } \\
\text { cerebellum, cortex }\end{array}$ \\
\hline 3 & $111,148,711$ & SFPQ & Circadian cycle and behavior & $\begin{array}{l}\text { Brain, cortex, skin, } \\
\text { thyroid }\end{array}$ \\
\hline 3 & $110,788,891$ & NCDN & Learning and pigmentation & Cortex, brain, \\
\hline
\end{tabular}

JABG Manuscript Proof 
Supplementary figure 1 Whole-genome scan of the Kullback-Leiber divergence (KLD) and the locus specific component (alpha) results obtained with the SelEstim, and Bayescan software's respectively. The gray lines indicate the thresholds of significance for each approach. Genomic coordinates and statistical significance are plotted in the $x$ - and $y$ axis, respectively.

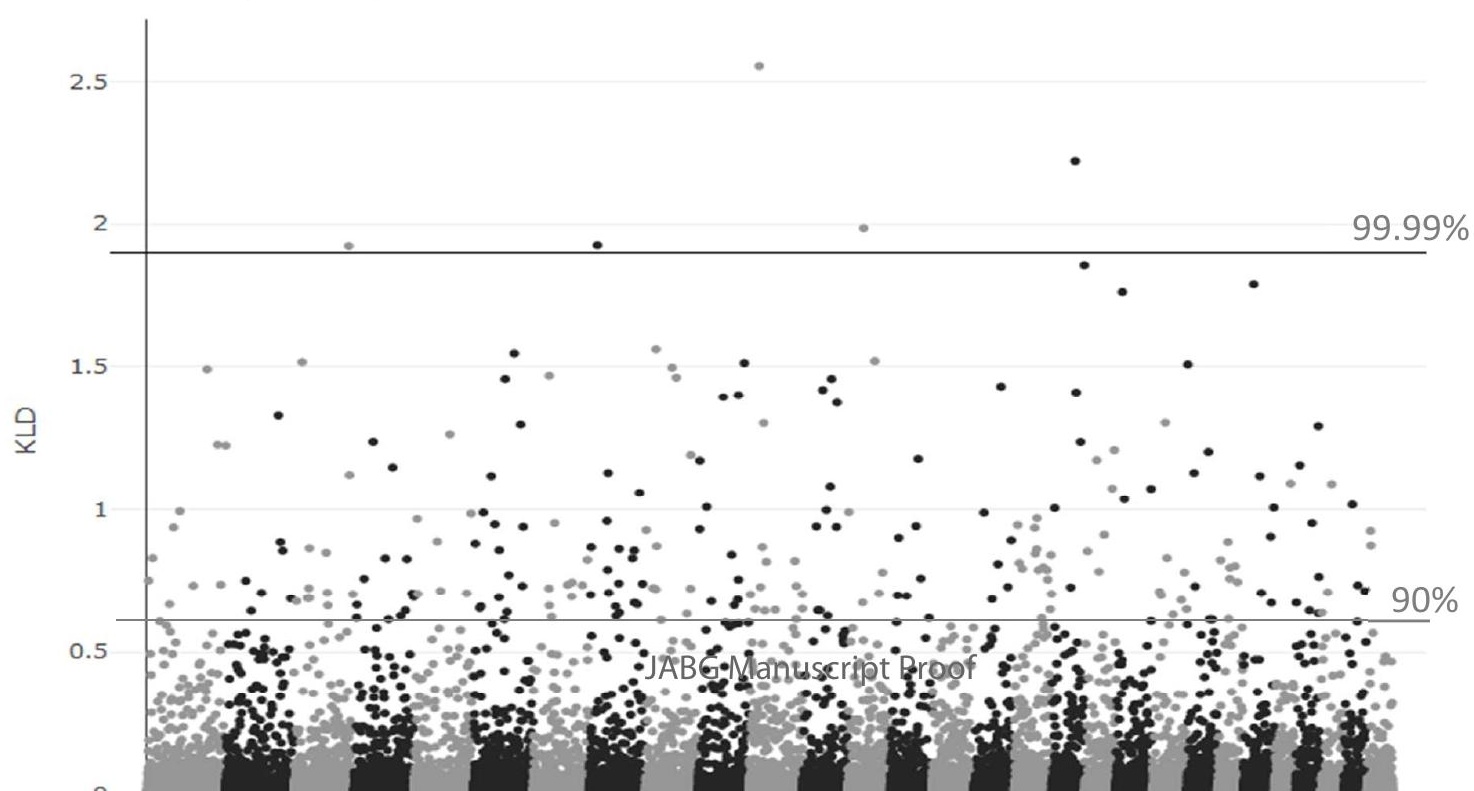

Supplementary table 4 Positional concordance between the SNPs detected at the 90\%KLD quantile of SelEstim and previous studies. Regions were constructed under the same selection criteria, using $10 \mathrm{Mb}$ sliding window spans. Chr: chromosome, Reg $(\mathrm{Mb})$ : region in $\mathrm{Mb}, \mathrm{N}$ SNP: number of markers with the higher value of $K L D$, and its positional concordance with previously reported regions detected on Spanish cattle breeds.

\begin{tabular}{lccccc}
\hline Chr & Reg (Mb) & N SNP & Higher KLD & $\begin{array}{c}\text { González-Rodriguez } \\
\text { et } \boldsymbol{a l . 2 0 1 6}\end{array}$ & $\begin{array}{c}\text { González-Rodriguez } \\
\text { et } \boldsymbol{a l . 2 0 1 7}\end{array}$ \\
\hline 2 & $0.24-7.24 *$ & 9 & 0.52 & $1.04-11.89 \mathrm{Mb}$ & $5.57-10.15 \mathrm{Mb}$ \\
6 & $29.55-37.74$ & 6 & 1.11 & $37.85-41.16 \mathrm{Mb}$ & $37.85-39.44 \mathrm{Mb}$ \\
7 & $42.62-51.57$ & 15 & 0.49 & $47.27-47.75 \mathrm{Mb}$ & $47.40-47.50 \mathrm{Mb}$ \\
11 & $65.25-74.35$ & 7 & 0.52 & $65.07-72.20 \mathrm{Mb}$ & $67.05-67.08 \mathrm{Mb}$ \\
13 & $56.53-66.18$ & 13 & 0.71 & $57.43-57.75 \mathrm{Mb}$ & $57.58 \mathrm{Mb}$ \\
18 & $4.90-13.45$ & 14 & 1 & $12.68-16.20 \mathrm{Mb}$ & $13.34-15.24$ \\
\hline
\end{tabular}

${ }^{*}$ The MSTN gene (myostatin) is located within this region 6.213.566..6.220.196 bp

$\begin{array}{lll}111,521,856 & \text { GJB4 } & \text { Olfactory neurophysiology } \\ 113,649,002 & \text { SAG } & \text { Visual stimulus neurophysiology } \\ 114,238,014 & \text { TRPM8 } & \text { Thermoception } \\ 108,509,996 & \text { POU3F1 } & \text { Neurogenesis } \\ & & \text { Muscle contraction } \\ 108,551,386 & \text { FHL3 } & \text { Neuronal disorders } \\ 15,681,923 & \text { LINGO2 } & \text { Neurodegenerative diseases }\end{array}$

cerebellum

Retina

Liver, prostate cortex, brain

Skeletal muscle, tibial

Brain, cortex, thyroid, uterus, testis

Brain, cortex, thyroid, skin, testis

* mRNA expression in normal human tissues from GTEx, Illumina Body map 